\section{POS0803 TRENDS IN THE INCIDENCE OF GIANT CELL ARTERITIS ACROSS SEVEN DECADES AND CHANGES IN DIAGNOSTIC MODALITIES: A POPULATION-BASED STUDY}

T. Garvey ${ }^{1}$, C. S. Crowson ${ }^{1}$, M. Koster ${ }^{1}$, K. J. Warrington ${ }^{1}{ }^{1}{ }^{M}$ Mayo Clinic, Rheumatology, Rochester, United States of America

Background: Diagnostic methods for giant cell arteritis (GCA) have evolved over recent decades, and large vessel imaging plays an increasing role in disease detection.

Objectives: This study aims to estimate the incidence of GCA over the past 10 years in a population and compare it to preceding incidence estimates. It also explores trends in the diagnostic modalities used to identify GCA.

Methods: A pre-existing population-based cohort of patients diagnosed with GCA between 1950 and 2009 was extended with incident cases from 2010 to 2019. The diagnosis of GCA was confirmed by review of medical records of patients with ICD9/10 codes for GCA between 1/1/2010 and 12/31/2019. Incident cases that met either one of the following sets of inclusion criteria were added to the cohort: one, American College of Rheumatology 1990 GCA classification criteria; or two, patients aged $\geq 50$ years with elevation of erythrocyte sedimentation rate or C-reactive protein and radiographic evidence of large vessel vasculitis attributed to GCA. Incident cases were classified into one of three groups: group 1, temporal artery biopsy (TAB) positive; group 2, TAB negative or not done with positive large-vessel imaging; or group 3, clinical diagnosis of GCA.

Results: The study cohort included 305 patients diagnosed with GCA from 1950 until 2019. Fifty-five incident cases were diagnosed between 2010 and 2019; 37 females $(67 \%)$ and 18 males (33\%). The age and sex adjusted incidence rates $(95 \% \mathrm{Cl}$ ) per 100,000 between 2010 and 2019 for females, males, and the total population were $13.0(8.8,17.3), 8.6(4.6,12.7)$, and $10.8(8.0,13.7)$, respectively. The corresponding incidence rates from $2000-2009$ were $28.0(21.0,35.1)$, $10.2(5.0,15.5)$, and $20.5(15.9,25.1)$, respectively. This represents a significant decline in the incidence rates in females $(p<0.001)$ and the total group $(p<0.001)$ between the 2000-2009 and 2010-2019 cohorts but no change in males $(p=0.64)$. Of the 55 patients diagnosed between 2010 and 2019, there were $37(67 \%)$ in group 1, $10(18 \%)$ in group 2, and $8(15 \%)$ in group 3. In contrast, of the 250 patients diagnosed between 1950 and 2009 there were $209(84 \%)$ in group 1, $4(2 \%)$ in group 2, and $37(15 \%)$ in group 3 . There was a significant difference between the 1950-2009 and 2010-2019 cohorts in the composition of these groups $(\mathrm{p}<0.001)$.

Conclusion: In this population-based cohort of patients with GCA diagnosed over a 70-year period, the incidence of GCA has declined in recent years. The total decline is driven by a decline in females but not in males. The reasons for this are unclear but should be followed over time and investigated in other population-based cohorts. There has also been a shift in the diagnostic modalities for GCA. In recent years, there are fewer TAB positive patients, and more patients diagnosed with large vessel imaging. This is the first population-based incidence cohort demonstrating a trend towards increased use of large vessel imaging for the diagnosis of GCA.

REFERENCES:

[1] Chandran AK, et al. Incidence of Giant Cell Arteritis in Olmsted County, Minnesota, over a 60-year period 1950-2009. Scand J Rheumatol. 2015;44(3):215-218.

[2] Gonzalez-Gay MA, et al. Giant cell arteritis: is the clinical spectrum of the disease changing? BMC Geriatr. 2019; Jul 29;19(1):200.

[3] Rubenstein E, et al. Sensitivity of temporal artery biopsy in the diagnosis of giant cell arteritis: a systemic literature review and meta-analysis. Rheumatology (Oxford). 2020 May 1:59(5):1011-1020.

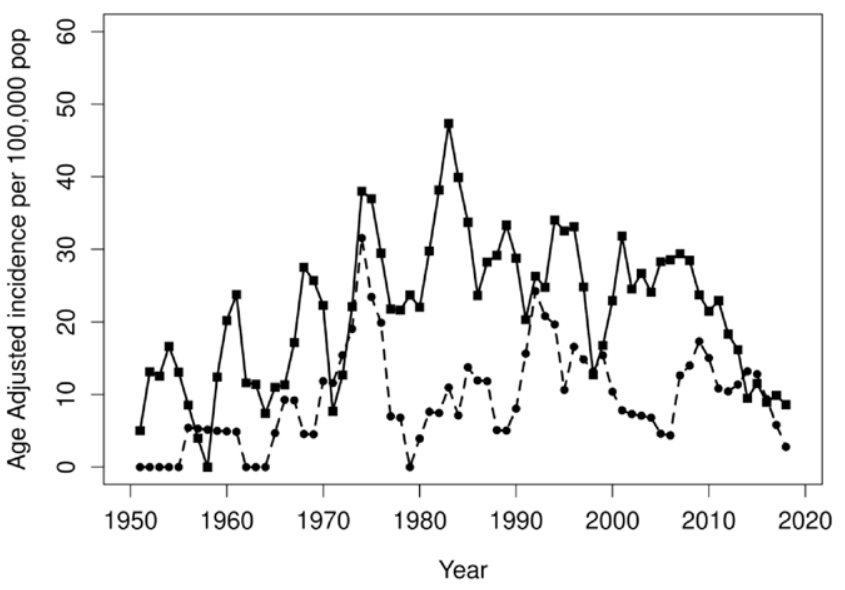

Figure 1. Trends in the incidence of GCA in Olmsted County by sex (1950-2019).
Acknowledgements: This study was made possible using the resources of the Rochester Epidemiology Project, which is supported by the National Institute on Aging of the National Institutes of Health $(\mathrm{NIH})$ under Award Number R01 AG034676, and CTSA Grant Number UL1 TR000135 from the National Center for Advancing Translational Sciences (NCATS), a component of the NIH. The content is solely the responsibility of the authors and does not necessarily represent the official views of the NIH.

Disclosure of Interests: Thomas Garvey: None declared, Cynthia S. Crowson: None declared, Matthew Koster: None declared, Kenneth J Warrington Grant/ research support from: Clinical research support from Eli Lilly and Kiniksa DOI: 10.1136/annrheumdis-2021-eular.1415

\section{\begin{tabular}{|l|l|l|}
\hline POS0804 OUTCOMES OF SURGICALLY RESECTED THORACIC & \\
\hline
\end{tabular} AORTITIS: RESULTS FROM A PROSPECTIVE MULTI- CENTRE REGISTRY}

H. Caterson ${ }^{1}$, A. Li' ${ }^{2}$, L. March ${ }^{3}$, P. Bannon ${ }^{4}$, J. Kench ${ }^{5}$, T. Davidson ${ }^{6}$, H. C. Soh ${ }^{7}$, A. Sammel ${ }^{8}{ }^{1}$ Royal Prince Alfred Hospital, Rheumatology, Camperdown, Australia; ${ }^{2}$ The University of Sydney, Charles Perkins Centre and Boden Institute, Camperdown, Australia; ${ }^{3}$ Royal North Shore Hospital, Rheumatology, St Leonards, Australia; ${ }^{4}$ Royal Prince Alfred Hospital, Cardiothoracic Surgery, Camperdown, Australia; ${ }^{5}$ Royal Prince Alfred Hospital, Anatomical Pathology, Camperdown, Australia; ${ }^{6}$ Prince of Wales Hospital, Anatomical Pathology, Randwick, Australia; ${ }^{7}$ Royal North Shore Hospital, Anatomical Pathology, St Leonards, Australia; ${ }^{8}$ Prince of Wales Hospital, Rheumatology, Randwick, Australia

Background: Inflammatory thoracic aortitis is a cause of aneurysm and is often diagnosed incidentally following aortic replacement surgery. It may be due to systemic diseases such as giant cell arteritis (GCA) and Takayasu arteritis (TAK) or a topographically isolated variant termed clinically isolated aortitis (CIA). While $\mathrm{CIA}$ is identified in $2-12 \%$ of thoracic aortic surgical pathology, ${ }^{1}$ its short-term post-operative outcome is unknown.

Objectives: This study was undertaken to examine the short-term post-operative mortality and morbidity of patients with surgically resected thoracic aortitis compared to those with non-inflammatory thoracic aortic aneurysms and assess if outcomes varied based on post-operative corticosteroid therapy.

Methods: The study was based at three tertiary referral hospitals in Sydney, Australia, for the years 2004-2018. Prospectively collected data was accessed from a national surgical registry for all patients who underwent thoracic aortic aneurysm replacement. Aortitis patients were identified from histopathology records.

Inpatient medical records were reviewed to categorise aortitis cases as $\mathrm{CIA}$, GCA, TAK or other aortitis and to audit the use of corticosteroids. Outcomes were compared between aortitis cases and the non-inflammatory cohort using logistic regressions, controlling for age, sex, year of procedure, and hospital.

Results: 41 aortitis cases were identified from the cohort of 1119 surgical patients (3.7\%). 27 (66\%) met criteria for CIA, 11 (27\%) for GCA, 2 (5\%) for TAK, and 1 (2\%) for Other. $8(20 \%)$ received corticosteroid therapy for aortitis.

Compared with non-inflammatory patients, the aortitis cohort was predominantly female ( $54 \%$ vs $28 \%, p<0.01$ ), older (mean 70 vs 62 years, $p<0.01$ ), and had higher rates of hypertension ( $83 \%$ vs $67 \%, p=0.03$ ) and pre-operative immunosuppression $(10 \%$ vs $1 \%, p<0.01)$

There was no difference $(p>0.05)$ between the aortitis and the non-aortitis groups for 30 -day mortality ( $7 \%$ vs $7 \%$ ), composite significant morbidity $(15 \%$ vs $22 \%$ ), composite infection (10\% vs $6 \%)$, return to theatre for bleeding $(7 \%$ vs $13 \%)$, stroke ( $5 \%$ vs $5 \%$ ) or readmission ( $12 \%$ vs $10 \%)$. Similar results were also seen for $\mathrm{ClA}$ and the aortitis subgroup who did not receive corticoid steroid therapy when compared to the non-aortitis group (table 1).

Table 1. 30-day mortality and morbidity

\begin{tabular}{|c|c|c|c|c|c|c|c|c|}
\hline & \multicolumn{2}{|c|}{ Non-aortitis } & \multicolumn{2}{|c|}{ All aortitis } & \multicolumn{2}{|c|}{$\begin{array}{l}\text { Aortitis, not } \\
\text { treated for } \\
\text { aortitis }\end{array}$} & \multicolumn{2}{|c|}{$\mathrm{ClA}$} \\
\hline & \multicolumn{2}{|c|}{$n=1078$} & \multicolumn{2}{|c|}{$\mathrm{n}=41$} & \multicolumn{2}{|c|}{$n=33$} & \multicolumn{2}{|c|}{$\mathrm{n}=27$} \\
\hline & $\%$ & $n$ & $\%$ & $\mathrm{n}$ & $\%$ & $\mathrm{n}$ & $\%$ & $\mathrm{n}$ \\
\hline Mortality & 7 & 70 & 7 & 3 & 6 & 2 & 4 & 1 \\
\hline Stroke & 5 & 57 & 5 & 2 & 6 & 2 & 4 & 1 \\
\hline Return to theatre bleed & 13 & 137 & 7 & 3 & 6 & 2 & 4 & 1 \\
\hline Composite morbidity & 22 & 241 & 15 & 6 & 15 & 5 & 11 & 3 \\
\hline Composite infection & 6 & 69 & 10 & 4 & 9 & 3 & 11 & 3 \\
\hline Readmission & 10 & 108 & 12 & 5 & 12 & 4 & 11 & 3 \\
\hline
\end{tabular}

Note: Differences in outcomes between the aortitis groups and the no aortitis group were modelled using logistic regression, controlling for age, sex, year of procedure, and hospital. There were no statistically significant differences in outcomes $(p>0.05)$.

$\mathrm{CIA}$; clinically isolated aortitis. Composite morbidity; an outcome comprised of return to the atre for bleeding, stroke, new renal failure, acute myocardial infarction or aortic dissection Composite infection; an outcome comprised of 30-day pneumonia, septicaemia, deep sterna wound infection or deep thoracotomy infection. 
Conclusion: The finding of thoracic aortitis following aortic replacement surgery does not affect short-term post-operative mortality or morbidity. Corticosteroid therapy in the immediate post-operative period did not impact upon short-term outcomes.

\section{REFERENCES:}

[1] Quimson L, Mayer A, Capponi S, et al. Comparison of aortitis versus noninflammatory aortic aneurysms among patients who undergo open aortic aneurysm repair. Arthritis Rheum 2020;72:1154-59.

Disclosure of Interests: None declared

DOI: 10.1136/annrheumdis-2021-eular.1531

\section{POS0805 \\ TREATMENT OF GIANT CELL ARTERITIS WITH TOCILIZUMAB: A RETROSPECTIVE COHORT STUDY OF 119 PATIENTS}

J. Rakholiya ${ }^{1}$, M. Koster ${ }^{1}$, H. Langenfeld ${ }^{2}$, C. S. Crowson ${ }^{1,2}$, A. Abril ${ }^{3}$, P. Bansal ${ }^{4}$, L. Mertz ${ }^{5}$, A. Rodriguez-Pla ${ }^{5}$, R. Sehgal ${ }^{4}$, B. Wang ${ }^{3}, K$. J. Warrington ${ }^{1}{ }^{1}$ Mayo Clinic, Rheumatology, Rochester, United States of America; ${ }^{2}$ Mayo Clinic School of Health Sciences, Biomedical Statistics and Informatics, Rochester, United States of America; ${ }^{3}$ Mayo Clinic, Rheumatology, Jacksonville, United States of America; ${ }^{4}$ Mayo Clinic Health System, Rheumatology, Eau Claire, United States of America; ${ }^{5}$ Mayo Clinic, Rheumatology, Scottsdale, United States of America

Background: Giant cell arteritis (GCA) is an inflammatory condition of mediumand large-sized arteries. Prospective clinical trials have demonstrated the efficacy of tocilizumab (TCZ) for treatment of patients with GCA (1). However, there is a limited data on the use of TCZ in routine clinical practice.

Objectives: To evaluate the efficacy and safety of TCZ in a retrospective cohort study of patients with GCA treated with TCZ

Methods: Patients with GCA treated with TCZ at 4 clinical centers of a single tertiary care institution (2000-2020) were identified. The diagnosis of GCA was confirmed by at least one of the following modalities: 1. Arterial biopsy 2. Large vessel imaging 3. Clinical diagnosis of GCA meeting ACR classification criteria and established by a rheumatologist. Patient demographics, clinical presentation, laboratory studies, treatment course and adverse events were abstracted from the medical record; only patients with at least 6 months of follow-up after TCZ initiation were included. Kaplan-Meier methods were used to estimate time to TCZ discontinuation and time to first relapse after TCZ discontinuation. Poisson regression models were used to compare relapse rates before and after TCZ initiation.

Results: The study cohort included 119 patients [61\% female; mean (SD) age at GCA diagnosis 70.3 (8.2) years]. The majority of patients $(89 \%)$ had a biopsy-proven and/or imaging-based diagnosis of GCA, while $13(11 \%)$ had a clinical diagnosis of GCA. In addition to glucocorticoids, 40 (34\%) patients received other immunosuppressive agents prior to TCZ. The method of initial TCZ administration was subcutaneous $(162 \mathrm{mg} / \mathrm{ml})$ weekly in $48(41 \%)$, subcutaneous every other week in 20 (17\%), monthly $4 \mathrm{mg} / \mathrm{kg}$ infusions in $34(29 \%)$, monthly $8 \mathrm{mg} / \mathrm{kg}$ infusions in $14(12 \%)$ and non-standard dosing in 3 remaining patients. The median (IQR) duration from GCA diagnosis to TCZ initiation was 4.8 (1.2-22.0) months and the median (IQR) duration of TCZ treatment was 18 (11-28) months. The mean (SD) dose of prednisone at TCZ initiation was 31 (19) $\mathrm{mg}$ /day and was reduced to a mean (SD) dose of 3.9 (6.7) $\mathrm{mg} /$ day at TCZ discontinuation/last follow-up visit. The relapse rate per year decreased $43 \%$ from 0.77 to 0.44 after the initiation of TCZ (RR=0.57; $95 \% \mathrm{Cl}: 0.44-0.75$; $\mathrm{p}<0.001)$. The mean (SD) ESR and CRP decreased from 22 (20) $\mathrm{mm} / \mathrm{hour}$ to $6(9.2) \mathrm{mm} /$ hour and from 19.1 (25) $\mathrm{mg} / \mathrm{L}$ to 5.4 (16.6) $\mathrm{mg} / \mathrm{L}$, respectively from TCZ initiation to TCZ discontinuation/last follow-up visit. At 2 years of follow-up, $67 \%$ of patients had discontinued glucocorticoids. At last follow up, 46 patients had discontinued TCZ, only 14 of which were due to adverse events. The median time to TCZ discontinuation was 2.9 years. Only $17 \%(95 \% \mathrm{Cl}$ : $10-24 \%)$ had discontinued by 1 year after TCZ initiation and 38\% $(95 \% \mathrm{Cl}$ : $26-47 \%$ ) had discontinued by 2 years. The most common adverse events were infections and cytopenias. While on TCZ, 1 patient developed new onset vision loss related to GCA and 1 patient, without history of diverticulitis, had bowel perforation. Among those discontinuing TCZ, 61\% had relapsed at least once by 1 year after TCZ discontinuation.

Conclusion: In this large single institution cohort of patients with GCA, TCZ use resulted in a significantly reduced relapse rate and reduction in glucocorticoid dosage. Overall, patients tolerated long-term use with only $12 \%$ discontinuing due to adverse events. However, over half of patients stopping TCZ had a subsequent flare; highlighting ongoing use may be required beyond two years in several patients with GCA to maintain remission. REFERENCES

[1] Stone JH, et al. Trial of Tocilizumab in Giant-Cell Arteritis. N Engl J Med. 2017 Jul 27;377(4):317-328. doi: 10.1056/NEJMoa1613849. PMID: 28745999.
[2] Calderón-Goercke M, et al. Tocilizumab in giant cell arteritis. Observational, open-label multicenter study of 134 patients in clinical practice. Semin Arthritis Rheum. 2019 Aug;49(1):126-135. doi: 10.1016/j.semarthrit.2019.01.003. Epub 2019 Jan 5. PMID: 30655091.

Disclosure of Interests: Jigisha Rakholiya: None declared, Matthew Koster: None declared, Hannah Langenfeld: None declared, Cynthia S. Crowson: None declared, Andy Abril: None declared, Pankaj Bansal: None declared, Lester Mertz: None declared, Alicia Rodriguez-Pla: None declared, Rahul Sehgal: None declared, Benjamin Wang: None declared, Kenneth J Warrington Grant/research support from: Research support: Kiniksa, Eli Lilly

DOI: 10.1136/annrheumdis-2021-eular.1674

\section{POS0806 FINDINGS CONSISTENT WITH SUBCLINICAL VASCULITIS IN PATIENTS WITH NEW ONSET POLYMYALGIA: A SYSTEMATIC LITERATURE REVIEW AND A META-ANALYSIS OF COHORT DATA}

D. S. Gozzoli ${ }^{1}$, A. Hemmig ${ }^{2}$, L. Hemkens ${ }^{3,4,5}$, L. Werlen ${ }^{3}$, H. Ewald ${ }^{6}$, C. Berger ${ }^{7}$, D. Kyburz ${ }^{2,8}$, S. Imfeld ${ }^{9}$, M. Aschwanden ${ }^{9}$, M. Stegert $^{2}$, D. Camellino ${ }^{10}$, M. A. Cimmino ${ }^{11}$, C. Campochiaro ${ }^{12}$, A. Tomelleri $^{12}$, L. Henckaerts ${ }^{13}$, D. Blockmans ${ }^{13}$, P. Moya ${ }^{14}$, H. Corominas ${ }^{15}$, R. Buchanan ${ }^{16,17}$, C. Owen ${ }^{16,17}$, Y. Van Sleen ${ }^{18}$, E. Brouwer ${ }^{18}$, H. Ymashita ${ }^{19}$, T. Daikeler ${ }^{2} .{ }^{1}$ University Hospital of Basel, Department of Internal Medicine, Basel, Switzerland; ${ }^{2}$ University Hospital of Basel, Department of Rheumatology, Basel, Switzerland; ${ }^{3}$ University Hospital of Basel, Department of Clinical Research, Basel, Switzerland; ${ }^{4}$ Stanford University, Meta-Research Innovation Center at Stanford (METRICS), Palo Alto, CA 94305, United States of America; ${ }^{5}$ Berlin Institute of Health, Meta-Research Innovation Center Berlin (METRIC-B), Berlin, Germany; ${ }^{6}$ University of Basel, Medical Library, Basel, Switzerland; ${ }^{7}$ University Hospital of Basel, Departments of Biomedicine and Internal Medicine, Translational Immunology and Medical Outpatient Clinic, Basel, Switzerland; ${ }^{8}$ University of Basel, Department of Biomedicine, Basel, Switzerland; ${ }^{9}$ University Hospital of Basel, Department of Angiology, Basel, Switzerland; ${ }^{10}$ "La Colletta"Hospital, Division of Rheumatology, Arenzano, Italy; ${ }^{11}$ University of Genoa, Research Laboratory and Academic Division of Clinical Rheumatology, Department of Internal Medicine, Genoa, Italy; ${ }^{12}$ San Raffaele Scientific Institute, Unit of Immunology, Rheumatology, Allergy and Rare Diseases (UnIRAR), Milan, Italy; ${ }^{13} \mathrm{KU}$ Leuven, University Hospitals Leuven, Department of General Internal Medicine, Department of Microbiology, Immunology and Transplantation, Leuven, Belgium $;{ }^{14}$ Hospital de la Santa Creu i Sant Pau, Rheumatology Unit, Department of Internal Medicine, Barcelona, Spain; ${ }^{15}$ Hospital Universitari de la Sant creu i Sant Pau, Servei de Reumatologia, Barcelona, Spain;

${ }^{16}$ Austin Health, Department of Rheumatology, Heidelberg, Victoria, Australia; ${ }^{17}$ University of Melbourne, Department of Medicine, Parkville, Victoria, Australia; ${ }^{18}$ University of Groningen, University Medical Center Groningen, Groningen, Netherlands; ${ }^{19}$ National Center for Global Health and Medicine, Division of Rheumatic Diseases, Tokyo, Japan

Background: GCA is characterized by cranial symptoms but imaging techniques show that patients with non-specific symptoms such as systemic inflammation or PMR may have undiagnosed large vessel (LV) GCA ${ }^{1}$. Although silent GCA in patients with clinically isolated PMR may have consequences for patients' outcome, little is known about its prevalence and characteristics of affected patients.

Objectives: To review data on the prevalence of silent GCA in newly diagnosed PMR patients without cranial GCA symptoms and to analyze which characteristics are associated with vascular involvement among PMR patients.

Methods: We systematically screened PubMed, Embase and Web of Science databases and included studies screening for GCA in steroid naïve PMR patients without cranial symptoms consistent with GCA. Authors of the publications that used PET for vasculitis screening were invited to share their individual patient data (IPD) for a meta-analysis. We sought to define patient characteristics that were associated with vasculitis using univariable mixed effects logistic regression models with vascular involvement as the outcome, missing values were imputed using multilevel joint modeling multiple imputation. To fit a multivariable mode with the candidate predictors we excluded variables that were hypothesized to have less medical relevance for the outcome and highly correlated inflammation markers (ESR, Lc).

Results: Out of the 3047 studies screened independently by 2 authors (DG and TD), 13 fulfilled the inclusion criteria. These studies (published 1963-2019) reported on 543 PMR patients examined by temporal artery biopsy $(n=175)$, ultrasound $(n=110)$, PET or PET-CT $(n=258) .115$ PMR patients were diagnosed with GCA (21.2\%), with prevalence ranging from $0-92 \%$.

We collected IPD for 243 patients from 4 cohorts using PET and 3 using PET/ CT for GCA diagnosis. The overall median age of patients was 72.3 years (IQR 66.4-78.0) and vasculitis was found in 65 patients (26.7\%) (table 1). 